\title{
Les diliférents types de protection contre la corrosion mis en ceuvre à l'usine marémotrice de la Rance
}

\author{
par M. Faral \\ E.D.F., Direction de l'Equipement
}

Parmi les problèmes qui se sont posés, dès que le projet de l'usine marémotrice de la Rance a pris corps, celui de la résistance à la corrosion du matériel, qu'il soit immergé, en marnage, ou exposé à l'atmosphère saline, s'est vite révélé important et délicat.

Il s'agissait de choisir dans l'arsenal existant ou de promouvoir des matériaux et des procédés de mise en œuvre capables d'assurer, sinon la pérennité du matériel, du moins son maintien en bon état de fonctionnement, moyennant des dépenses d'entretien qui ne soient pas significativement différentes de celles d'une installation similaire travaillant en eau douce. La conception de départ ne devait toutefois pas alourdir de façon exorbitante le coût initial d'investissement.

Assurément, l'expérience de matériels travaillant en immersion dans l'eau de mer était importante, qu'il s'agisse d'ouvrages fixes tels que les écluses ou de la construction navale. Toutefois, cette dernière s'accommodait d'immobilisations pour entretien sensiblement plus élevées que celles qui étaient usuelles en matière d'équipements hydroélectriques, où, lorsqu'il n'y a pas de possibilités d'accumulation à long terme comme dans le cas considéré, le coût des pertes d'énergie pour entretien dépasse de beaucoup celui de l'entretien lui-même.

Ce n'est pas l'objet de cette communication que de faire l'historique des nombreuses recherches qui ont précédé et permis d'éclaircir le choix qu'il a fallu faire au moment de passer à la réalisation. Il s'agit seulement de rappeler les options prises et d'indiquer brièvement ce qu'on attendait d'elles. Les communications suivantes auront pour but d'exposer si, après cinq années d'exploitation, les résultats obtenus permettent de dire que le but recherché a été ou non atteint, ou s'ils amènent à rester encore dans l'expectative. Il paraissait également tentant, et ceci fera l'objet de la dernière communication, d'examiner si l'acquis des douze dernières années - car si l'exploitation a commencé il y a cinq ans, les techniques de réalisation ont été pratiquement figées depuis 1960 - permettrait, à supposer que le début des travaux soit aujourd'hui, de retenir des solutions plus satisfaisantes que celles choisies à l'époque.

$$
\%
$$

Le matériel en contact avec l'eau de mer, de façon permanente ou en marnage, comprend, pour l'essentiel, des turbines, des vannes (parties fixes et mobiles), des portes d'écluses, un réseau de tuyauteries (vidange, exhaure, lubrification des joints d'arbre), la robinetterie et les pompes correspondantes. Le degré de sévérité de l'attaque par corrosion est sensiblement le même pour ces différents matériels.

Par contre, le degré de contact avec l'air salin, c'est-à-dire avec un air plus ou moins chargé de fines vésicules d'eau de mer qui viennent, le cas échéant, se déposer et sécher sur les parois, est nuancé selon qu'il s'agit de charpentes ou de portiques de vannes directement exposées aux intempéries sur le couronnement du barrage, ou de l'ensemble des matériels situés à l'intérieur de la centrale, depuis les transformateurs principaux jusqu'au petit appareillage électrique de commande et de contrôle.

Un premier ensemble de mesures, pour se défendre contre la corrosion, consistait à l'évidence à isoler le matériel du milieu agressif, là où ce contact ne résultait pas du fonctionnement même de l'installation.

C'est ainsi que l'eau de mer a été proscrite comme agent réfrigérant, sauf à trouver comme paroi froide des échangeurs une surface qui, pour d'autres raisons, serait en tout état de cause en contact avec celle-ci: c'est le cas des 
avant-distributeurs des turbines, structures caissonées creuses immergées dans le courant, et à l'intérieur desquelles circule et se refroidit l'huile de graissage.

Lorsque des structures immergées étaient assemblées par des brides ou des systèmes similaires, pour des questions de démontabilité, des joints élastiques limitaient la pénétration de l'eau entre les faces d'appui à la profondeur minimum, la boulonnerie de liaison étant ainsi mise hors d'eau.

Dans le même ordre d'idée, l'air nécessaire à la ventilation de la centrale a été prélevé assez loin du rivage, en distance et en altitude, pour minimiser l'introduction dans celle-ci de particules salines. L'appareillage électrique délicat a été enfermé dans des armoires qui «respirent», mais à travers un système de chicanes chauffées, destinées à piéger les particules d'eau salée en suspension dans l'atmosphère.

Un deuxième ensemble de mesures consistait, dès lors que le contact avec le milieu corrosif était inévitable, à adopter pour les surfaces intéressées une géométrie apte à rendre plus facile la résistance à la corrosion. Les états de surface réguliers, l'absence de replis, de recoins facilitent, quel que soit le type de protection adoptée, sa mise en œuvre, son efficacité en service, ou ces deux facteurs simultanément.

Toutefois, l'ensemble de ces dispositions constructives ne permettent que de limiter le champ d'action de la corrosion et d'en adoucir, dans une certaine mesure, l'agressivité. Le problème de fond restait le choix, pour les groupes, les vannes, et l'écluse, de matériaux et de procédés de protection bien adaptés.

Compte tenu des conditions de fonctionnement, le même matériel, travaillant en eau douce, aurait pu être réalisé en acier au carbone, ou en acier faiblement allié pour certaines pièces mécaniquement plus sollicitées. Toutefois, pour certaines parties actives de la turbine, la résistance à la cavitation aurait impliqué, en tout état de cause, l'emploi d'alliages résistant mieux à ce type de dégradations. De même, pour les vannes, des pièces fixes d'étanchéité n'auraient pu, en eau douce, être exécutées en acier au carbone.

Les différences de réalisation par rapport à ce qu'on pourrait appeler la solution «eau douce» peuvent se classer comme suit :

- emploi de matériaux de construction de même nature, acier ordinaire essentiellement, mais protégés par un revêtement de peinture, d'une qualité choisie après de nombreux essais;

- emploi de matériaux aptes en eux-mêmes à résister à la corrosion: acier à forte teneur en chrome et alliages cuivreux, matériaux à base de résines synthétiques,

- emploi d'une protection cathodique, à courant imposé (sauf quelques éléments qui sont protégés par des anodes sacrificielles), qui portent le métal à protéger dans la zone des potentiels de passivité

Il existe d'ailleurs une interaction entre ces différentes protections, qui fait qu'elles se complètent et qu'on ne saurait dire que tel élément a été protégé par peinture, l'autre par le choix du métal, le dernier par protection cathodique. C'est ainsi que la protection cathodique, appliquée à une grande surface non peinte, exige, pour obtenir le potentiel désiré, des intensités importantes qui posent des problèmes au niveau des anodes. La présence de peinture limite, par sa nature isolante, l'intervention de la protection cathodique aux zones où cette peinture a été détruite ou blessée. La présence simultanée d'acier ordinaire et d'acier fortement allié, ou d'alliage cuivreux, en contact galvanique, aggrave, par effet de couple, l'agressivité du milieu sur l'acier ordinaire. Le décalage de potentiel apporté par la protection cathodique permet de neutraliser cet inconvénient, mais déplace simultanément la plage normale de potentiel des aciers alliés.

$$
\text { * }
$$

La protection par peinture a été utilisée sur l'ensemble des parties en acier ordinaire, immergées ou non, des groupes, des vannes (que ce soient les vannes proprement dites du barrage, ou les vannes de batardage et de protection des groupes), de l'écluse.

De plus, là où devait exister en parallèle une protection cathodique, les parties en matériaux nobles ont été également revêtues avec le même système de peinture. Le but était de diminuer l'intensité anodique nécessaire qui, sans cette précaution, se serait répartie non seulement vers les surfaces accidentellement décapées de l'acier ordinaire mais aussi vers celles, volontairement laissées nues, des métaux nobles. Or, la répartition des intensités s'effectue au prorata des surfaces cathodiques, en tenant compte de la correction résultant des courbes de polarisation respectives de l'acier ordinaire et des métaux nobles. Seules ont fait exception les parties dont les conditions de fonctionnement ne permettaient pas d'espérer une tenue tant soit peu satisfaisante de la peinture, comme les pales de roue, les appuis d'étanchéité de vanne.

Le type de peinture retenu après les essais est un revêtement mince à base de résines vinyliques comme liant. Le revêtement comportait six couches: une couche de wash primaire réactive, trois couches primaires avec un pigment inhibiteur au chromate de zinc, deux couches de finitions inertes pour les parties hors d'eau, ou deux couches de finition actives antisalissures pour les parties immergées, le pigment étant, dans ce dernier cas, de l'oxydule de cuivre. Ces dernières couches sont consommables et leur efficacité est limitée dans le temps.

Un seul fournisseur de peinture fut retenu, le type de peinture qu'il proposait ayant montré, au cours d'une large campagne d'essais préliminaires, une tenue meilleure que celle des autres produits essayés. Cette supériorité était nettement marquée vis-à-vis d'autres types de revêtement, et sensible vis-à-vis de revêtements de même type, mais de marque différente.

On savait toutefois que les conditions d'application de ce type de protection sont très strictes et doivent être parfaitemient respectées si l'on veut retrouver à l'échelle industrielle, sans dégradation trop importante, les résultats observés sur les échantillons 'd'essai.

Une première précaution fut de demander aux constructeurs un état de surface ne présentant pas d'aspérités marquées, en tombant les angles vifs, en adoucissant par meulage l'état de surface des cordons de soudure. Toute irrégularité géométrique entraîne en effet une diminution locale de l'épaisseur du film déposé.

La deuxième mesure consista à créer, sur le site, un atelier d'application spécialisé. La totalité des pièces de groupes y ont été peintes ainsi que bon nombre d'autres structures métalliques.

Il était permis de penser que, grâce à une installation bien équipée et à une surveillance régulière, les résultats obtenus seraient supérieurs à ceux quauraient donnés une 
exécution dispersée chez des constructeurs, pour qui ce type de travail est, malgré tout, marginal. Le sablage, dont la bonne exécution est primordiale, a pu ainsi être exécuté dans d'excellentes conditions, le chauffage a permis de respecter le degré hygrométrique nécessaire à une application correcte.

Il semble donc que le maximum de précautions qui pouvaient être raisonnablement prises pour une exécution industrielle l'aient été. Cependant (et sans empiéter sur ce qu'il sera dit dans d'autres communications), les résultats n'ont pas été aussi satisfaisants qu'on l'avait espéré. Il fallait s'attendre à ce que la tenue dans le temps soit moins bonne que celle observée sur des échantillons d'essais, mais le décrochement a été nettement marqué. Comme ordre de grandeur, il pourrait être estimé à deux degrés de l'échelle européenne de degrés d'enrouillement.

Il a paru, en outre, très difficile d'obtenir, en cas de réfections in situ, la même qualité que celle obtenue au cours de la première exécution, et ceci est un handicap sérieux dès lors qu'on sait que cette réfection sera obligatoire au cours de la vie de l'installation.

Les observations ci-dessus sont essentiellement valables pour les surfaces immergées. Pour les surfaces soumises à l'air salin, les résultats peuvent être considérés comme très bons. Il a, dans ce cas, été parfois fait usage de la variante consistant à appliquer le même système, à une modification du wash primer près, sur des tôles en acier ordinaire préalablement zinguées, à chaud ou le plus souvent par voie électrolytique. Ce procédé a été employé notamment pour la tôlerie des armoires d'appareillage électrique.

Avant de quitter le domaine des peintures, et pour illustrer, à titre anecdotique, la régularité d'épaisseur que l'on peut obtenir en déposant les voiles successifs, on évoquera le problème de l'étanchéité des brides. Celle-ci, en règle générale, a été obtenue par le procédé gorge et joint torique en caoutchouc. Le système donne de bons résultats, moyennant un respect très strict des dimensions respectives de la gorge et du jonc. Les brides étant peintes, les gorges ont été préalablement usinées avec des surcotes correspondant à l'épaisseur que le film de peinture allait déposer sur les flancs et sur le fond de celles-ci, et l'étanchéité a été ainsi correctement obtenue.

$$
\because
$$

Les matériaux incorrodables par nature devaient être employés à bon escient, de par leur coût plus élevé. C'est d'ailleurs par un abus de langage que l'on parle de matériaux incorrodables dans la masse, tout au moins pour les métaux et alliages employés dans le cas présent, puisque l'effet de protection est dû à une couche de métal oxydé superficielle.

L'expression est toutefois correcte pour les résines synthétiques, qui ont été employées en petite quantité, essentiellement pour les tuyauteries de diamètre faible ou moyen.

Les trois résines utilisées, polychlorure de vinyle, polythène, époxy, résistent sans difficultés à l'eau de mer et les restrictions à leur emploi ont été dues à des caractéristiques mécaniques médiocres. Le polythène est souple, mais n'est guère utilisable au-delà de $40 \mathrm{~mm}$ de diamètre intérieur. Il a été utilisé pour des prises de pression, dont certaines sont noyées dans le béton de l'ouvrage, pour aller mesurer les hauteurs de chute. Le P.V.C. se trouve en diamètre plus important, mais il est fragile et résiste mal aux chocs mécaniques extérieurs et aux coups de bélier, et la rupture de la tuyauterie est alors totale. Il a été, de ce chef, prohibé pour les tuyauteries lorsque la mer était derrière, et réservé à des liaisons internes. Les tuyaux en résine époxy armés en fibre de verre n'ont été employés qu'à titre expérimental. Leur apparition sur le marché était récente, les accessoires de raccordement difficiles à trouver; leur raccordement, par emboîtement et collage, risquait de ne pas avoir la même résistance que celle du tube courant, qui est excellente et ne présente aucun des inconvénients indiqués plus haut. Pour peu que certains progrès aient été faits, ils doivent actuellement concurrencer très valablement les solutions métalliques.

Les pièces en métaux incorrodables étaient, soit des pièces moulées, soit des produits laminés (tôles essentiellement, quelques profilés, des tuyauteries de diamètre inférieur à $200 \mathrm{~mm}$ ). Le choix des nuances retenues, dans la famille des aciers au chrome d'une part, dans les alliages cuivreux d'autre part, était ainsi orienté par la nécessité de respecter, outre une bonne résistance à la corrosion, tout ou partie des conditions suivantes:

- résistance mécanique, statique ou à la fatigue, relativement élevée;

- bonne résistance à la cavitation;

- aptitude à la réparation par soudage pour les pièces sujètes à usure en exploitation;

- nuance susceptible d'être laminée.

Certaines des propriétés ci-dessus vont de pair, notamment la résistance à la cavitation et la résistance à la corrosion, et il n'y avait pas alors à rechercher un compromis entre deux propriétés contradictoires.

Pour les aciers au chrome, il y avait lieu de craindre essentiellement la corrosion par piqûre et la corrosion par aération différentielle. Pour cette dernière, on pouvait espérer la limiter en évitant les formes géométriques favorables à son apparition.

La teneur en chrome des nuances utilisées a été de $17 \%$ au minimum. Celles-ci comportaient en outre systématiquement une addition de molybdène, dont l'action bénéfique contre les piqûres est admise. On a utilisé essentiellement les nuances suivantes:

- Acier martensitique, de composition type suivante:

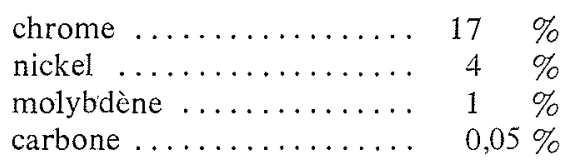

Cet acier a été utilisé sous forme moulée pour les pales, le moyeu de roue, le distributeur (directrices et anneaux) des turbines. Il a été utilisé sous forme laminée pour les galets et les rails de roulement des vannes wagon principales. C'est un acier qui permet de bonnes performances mécaniques, et la réparation par soudure des pièces moulées est relativement aisée.

- Acier austénitique au molybdène à très bas carbone. Cet acier, de composition classique, a été utilisé sous forme de tôles pour les manteaux de roue des turbines, pour les chemins de roulement des vannes de groupe, les portées d'étanchéité de celles-ci. Certaines tuyauteries ont été exécutées dans la même nuance.

- Acier austéno ferritique, au molybdène et à bas carbone. Il a été utilisé, sous forme de pièces moulées, avec 
une teneur en ferrite de lordre de $20 \%$, pour des chemises d'arbre et des labyrinthes de turbine.

En ce qui concerne les alliages cuivreux, les deux nuances principalement utilisées ont été des cupro aluminium. La première, uniquement sous forme moulée, pour les pales de roue a pour composition type:

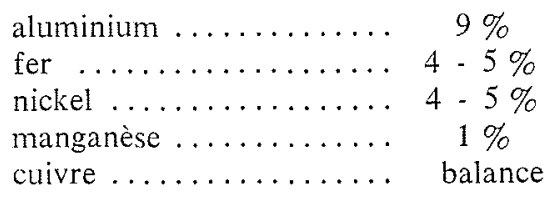

La seconde, qui peut se laminer, a été utilisée en tuyauterie - roulée-soudée, ou sans soudure pour les petits diamètres - Elle a pour composition type:

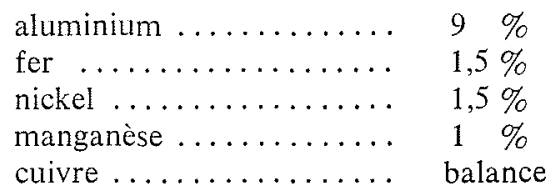

Cette dernière nuance a montré une certaine susceptibilité à la désaluminisation, et à la corrosion érosion. Par contre, la bonne résistance à la corrosion par aération différentielle des cupro-aluminium les a fait utiliser assez largement pour la boulonnerie immergée, de préférence aux aciers au chrome. A ce sujet, on peut ouvrir une parenthèse sur le problème de la boulonnerie immergée, qui n'a pas trouvé de solution satisfaisante. Les filets doivent etre au contact métal sur métal, ce qui interdit la peinture des parties filetées, et il reste une hélice creuse, où il est très difficile d'interdire à l'eau de pénétrer. L'emploi de métaux à potentiels différents pour la vis et l'écrou entraîne, dans les meilleurs délais, la destruction du filetage du métal le moins noble. Les aciers ordinaires rouillent et les aciers au chrome, en milieu confiné, ne se comportent guère mieux. Une des moins mauvaises solutions a été un vaselinage copieux des parties vissées, ce produit restant assez bien en place et étant plus stable dans le temps que les graisses.

En règle générale d'ailleurs, tout un ensemble de facteurs favorables au développement de la corrosion se trouvent réunis dès que les formes géométriques se compliquent. D'une façon un peu simpliste, on pourrait dire que le petit rayon de courbure est néfaste.

Enfin, la protection par métaux incorrodables, chromage essentiellement, n'a pas été employée en immersion, et avec parcimonie en exposition à l'air salin. Il est très difficile, en effet, que, tôt ou tard, une imperfection du revêtement ne permette l'attaque du métal sous-jacent et la destruction par en-dessous de la couche chromée.

$$
\text { : }
$$

Des options définies ci-dessus, il résultait qu'on avait à faire à deux types d'éléments, qui se jouxtaient ou s'imbriquaient: des éléments en acier ordinaire, protégés par peinture, et des éléments en alliages plus nobles, réputés se protéger eux-mêmes, mais simultanément aggraver les conditions de travail de lacier ordinaire, s'ils étaient en contact galvanique avec lui.

La première idée qui s'est fait jour, pour pallier cet inconvénient, a été d'insérer des joints isolants entre ces différents éléments, chacun travaillant de nouveau pour soi, et l'on supprimait ainsi les inconvénients du bimétallisme.
Un examen détaillé de la technologie à mettre en œuvre, pour réaliser cet isolement, montra rapidement qu'il s'agissait d'un problème difficile. Sur les groupes, le nombre de pièces à isoler était élevé, et le procédé d'isolation devait permettre la transmission d'efforts mécaniques importants, tout en respectant la précision de l'assemblage. Pour un assemblage par brides, par exemple, il fallait trouver un matériau apte à admettre la pression de serrage sans déformation, et conservant, dans le temps, ses qualités d'isolation. Il était difficile d'affirmer qu'un canon isolant ou une rondelle, mal placé ou oublié, ne serait pas la cause d'un court-circuit local, neutralisant l'efficacité du reste de l'isolation.

La difficulté d'une réalisation sûre de joints isolants a orienté vers l'utilisation d'une protection cathodique à courant imposé. L’idée initiale était de rattraper l'écart entre le potentiel d'équilibre des aciers ordinaires dans l'eau de mer et celui qu'ils auraient pris en contact avec des métaux plus nobles. En fait, comme il sera exposé dans les communications ultérieures, le rôle dévolu à cette protection a été très élargi, et elle agit en amenant dans la zone d'immunité les aciers ordinaires, sans que les aciers au chrome risquent de se trouver dans une zone dangereuse entre la passivité et l'immunité.

La protection cathodique a été, de construction, appliquée aux groupes. La difficulté inhérente à la protection cathodique de l'intérieur de toute surface fermée résulte du falt que les anodes sont quasi obligatoirement au niveau de la surface à protéger, donc à des distances très variables de celle-ci. Il s'ensuit que, si l'on veut obtenir une répartition assez régulière des densités de courant cathodique, il faut multiplier le nombre de points d'injection. C'est ainsi que chaque groupe a été équipé de trente-six anodes ponctuelles, dont la technologie a été soigneusement étudiée.

Les bons résultats obtenus ont incité à appliquer la protection cathodique aux vannes, puis à l'écluse, avec des anodes cette fois-ci beaucoup moins nombreuses, puisque c'est l'extérieur d'une surface qu'il s'agissait de protéger, et qu'on pouvait en écarter convenablement les anodes, mais l'exécution a été rendue plus difficile par l'obligation d'opérer dans des zones qui ne pouvaient plus être mises hors d'eau.

$$
*
$$

$\mathrm{Si}$, après ce rappel assez rapide de l'essentiel des dispositions prises contre la corrosion, on veut tenter de conclure, on pourra retenir que, dans ce domaine plus encore que dans un autre, il faut s'assurer de la représentativité des conditions d'essai, le milieu marin en particulier se diversifiant en fonction des lieux et des saisons.

Il faut également retenir qu'il n'existe pas de remède miracle, et qu'un comportement satisfaisant ne s'obtient que par l'addition d'un certain nombre de précautions qui s'épaulent et se complètent, et qui doivent être mises en œuvre de façon très méticuleuse.

Les opérations de mise en route, les premières années d'exploitation, ont montré que les faits se chargeaient d'un sérieux rappel à l'ordre, lorsque ces principes étaient transgressés.

\section{:}

Cette communication a été discutée en même temps que la communication suivante. On tronvera le texte de cette discussion en page 256. 